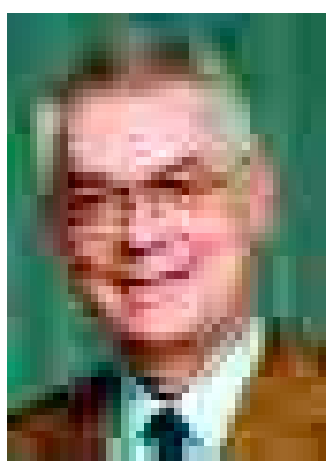

\title{
Ernährungsmedizin - Ein Stiefkind der heutigen Medizin
}

Es besteht Einigkeit darüber, dass in den entwickelten Ländern ein grosser Teil der Erkrankungen ernährungs(mit)bedingt ist. Besonders Adipositas, Diabetes mellitus und Hypertonie, die heutigen Geisseln der Wohlstandsgesellschaft, führen zu extrem hohen Folgekosten, die sich auch reiche Volkswirtschaften künftig kaum noch leisten können. Das Nahrungsangebot ist heute nahezu unbegrenzt, billig, hygienisch einwandfrei, oft schon zubereitet, praktisch in Verpackung und Portionierung, gut lagerfähig, von ubiquitärer Verfügbarkeit und hoher Energiedichte.

Die Therapie der Wohlstandskrankheiten beginnt meist erst bei Organmanifestierungen der Grunderkrankung und besteht vorwiegend aus medikamentösen Massnahmen. Da viele dieser Pharmaka die in sie gesetzten Erwartungen nicht erfüllen, werden immer neue und teure, innovative oder schein-innovative Arzneimittel auf den Markt geworfen, welche die eingetretene Entwicklung der Erkrankung rückgängig machen sollen.

Die für die Kosten der Krankheiten Verantwortlichen haben längst erkannt, dass die Wirksamkeit dieser neuen Medikamente nachgewiesen sein muss, bevor deren Kosten übernommen werden können. Diese Studien zum Nachweis der Wirksamkeit sind extrem kostenaufwendig. Sie können heute nur noch von leistungsstarken Konzernen in der Erwartung späterer hinreichender Erlöse aufgebracht werden.

An den Universitäten wird Ernährungsmedizin im Rahmen der Ausbildung von Ärzten praktisch nicht gelehrt und weniger noch geforscht. Da die Universitäten selbst nur über geringe Forschungsmittel verfügen, sind sie in hohem Masse auf die Einwerbung von Drittmitteln angewiesen. Diese werden meist von der Pharmaindustrie im Rahmen von Arzneimittelstudien angeboten, auf deren Ablauf und Auswertung die Hochschule wenig Einfluss hat. Die Hochschulmediziner beschränken sich auf das Vortragen dieser Ergebnisse auf Kongressen und Fortbildungsveranstaltungen. Da eine gesunde Ernährungsweise in der Regel nicht an ein kommerzielles Produkt gebunden ist, über das die Kosten einer Studie finanziert werden können, und öffentliche Mittel fehlen, gibt es nur wenige kontrollierte Studien, vergleichbar einer A rzneimittelstudie, welche die Wirksamkeit einer geeigneten Ernäh rungstherapie dieser Krankheiten belegen. Dennoch macht die Entwicklung dieser Krankheiten in Zusammenhang mit der Entwicklung des Wohlstands und des kommerziellen Nahrungsangebots den Zusammenhang evident.

Leider wird auch im Rahmen einer ärztlichen Praxis eine geeignete medizinische Ernährungsberatung, die viel Zeit erfordert und in der Regel nur mit geschultem Hilfspersonal (Diätassistenten oder Diplom-Oecotrophologen) betrieben werden kann, nicht hinreichend erstattet. Es ist erfreulich, dass das Wirken von Sven David Müller-Nothmann, der sich als Ernährungsberater und Sprecher der Gesellschaft für Ernährungsmedizin und Diätetik (GfED) e.V. seit vielen Jahren in vielen Beratungen, Schriften und Auftritten in den Medien, auch gegen Widerstände aus der Pharmaindustrie, verdient gemacht hat, mit dem Bundesverdienstkreuz anerkannt worden ist. Dennoch kann man sich mit einer solchen Ehrung nicht zufrieden geben. Es bedarf erheblicher Mittel, um in der Öffentlichkeit, in der Forschung und Ausbildung, in der ärztlichen Praxis das Bewusstsein für eine gesunde Ernährungsweise zu verbessern, um die nicht länger finanzierbaren Folgeerkrankungen einer Hyperalimentation zu mindern. Eine professionelle Ernährungstherapie gehört daher, auch wenn sie in der Schulmedizin wenig Interesse findet, zu der Grundausstattung einer modernen Medizin.

Prof. Dr. med. Helmut Mann

Wissenschaftlicher Direktor der Gesellschaft für Ernährungsmedizin und Diätetik e.V., DE-Aachen, info@ernaehrungsmed.de • www.ernaehrungsmed.de 